\title{
Règles de consentement pour l'utilisation d'échantillons biologiques à des fins de recherche dans le cadre de la HSCT en Suisse
}

\author{
Simone Romagnolia, Peter Bürklib, Jörg Halterc \\ a Assistant scientifique, PhD, Projet FNS, Université de Bâle \\ b Assistant scientifique, lic. iur., LL.M., Faculté de Droit, Université de Bâle \\ c Hématologue, MD, Hôpital universitaire de Bâle
}

La transplantation de cellules souches sanguines (hematopoïetic stem cell transplantation, HSCT) connaît d'importants changements, qui iront certainement en s'accentuant ces prochaines années suite à l'évolution des pratiques (comme, par exemple, l'injection de cellules souches directement dans la moelle osseuse ou la cotransfusion de cellules souches mésenchymateuses du sang du cordon) et aux applications attendues dans le domaine de la médecine régénérative (dans le traitement postinfarctus du myocarde ou l'ingénierie tissulaire de valvules cardiaques autologues, entre autres).

Pour permettre à ces changements de se réaliser dans l'intérêt des patients et dans le respect des droits et $\mathrm{du}$ bien-être des donneurs, il est important que la connaissance des facteurs déterminant le succès des HSCT puisse se baser sur des preuves scientifiquement validées que seule la recherche est en mesure d'apporter.

Dans cette optique, les donneurs sont au centre de l'attention des professionnels, puisqu'ils sont des acteurs indispensables mais vulnérables. C'est sur eux en effet que repose la pérennité de la HSCT; cependant, ils ne jouissent d'aucun bénéfice direct, mais sont exposés à des inconforts ou à des risques liés aux techniques de prélèvement. C'est pourquoi la World Marrow Donor Association (WMDA), l'organisation qui chapeaute et met en relation au niveau international 79 registres de donneurs et 156 banques de sang du cordon, élabore régulièrement des recommandations et fixe des standards pour assurer le respect des droits et le bien-être des donneurs [1].

Dans le cadre de la HSCT, le donneur peut être appelé à s'autodéterminer dans différentes situations - par exemple, au sujet de l'inscription dans le registre national des donneurs, du don avant le prélèvement (de moelle osseuse ou de cellules souches périphériques), de tests complémentaires, du prélèvement de lymphocytes, etc. -, mais curieusement aucun des onze formulaires utilisés actuellement au Service de transfusion CRS Suisse ne concerne spécifiquement la recherche. Pourtant, l'un des principaux défis auxquels la HSCT est confrontée, aujourd'hui et dans les prochaines années, consiste dans la cohabitation entre les activités diagnostiques et thérapeutiques d'une part, et les activités de recherche d'autre part, notamment l'implica- tion directe des donneurs dans des protocoles de recherche et l'utilisation d'échantillons de matériel biologique humain à des fins de recherche.

La WMDA s'est exprimée sur le premier défi [2], cependant le deuxième ne trouve pas encore de consensus au niveau international, il est donc important de s'y intéresser.

Il faut souligner que les échantillons de sang qui pourraient être utilisés à des fins de recherche n'ont généralement pas été prélevés dans cette optique; en effet, du matériel biologique subsiste après une transplantation ou un test d'histocompatibilité, et alimente ainsi les biobanques dans les centres de transplantation. Le donneur ne peut pas exprimer un consentement éclairé sur l'utilisation future de ces échantillons, parce qu'au moment du prélèvement ni le type de recherche ni les objectifs de celles-ci ne sont encore connus. Par ailleurs, il est évident que les échantillons conservés dans ces biobanques représentent une source précieuse pour la recherche. Il est donc impératif de définir les conditions légitimant leur utilisation à des fins de recherche, notamment en ce qui concerne la problématique du consentement.

Dans une directive de 2006 concernant les biobanques [3], l’Académie Suisse des Sciences Médicales suggère une issue à cette problématique en introduisant la règle du consentement général (broad consent, également blanket consent ou encore open consent). Au point 4.3 sur le consentement, on peut en effet lire que: «Le consentement peut se référer généralement à l'utilisation ultérieure des échantillons et données pour des projets de recherche futurs (consentement général), mais il peut aussi être limité à un domaine de recherche spécifique».

Les directives définissent trois conditions d'application de la règle du consentement général: 1) le prélèvement de matériel biologique humain à des fins de recherche ne constitue pas l'objectif premier; 2) les échantillons sont anonymisés; et 3) le prélèvement ne présente pas de risques particuliers pour le donneur. Relativement au champ d'application de ces directives, il est spécifié que: «l'utilisation éventuelle d'échantillons et de données après l'atteinte du premier objectif (par exemple après avoir arrêté un diagnostic) doit suivre ces directives.» 
On peut considérer que c'est justement par rapport à ce type d'utilisation que le consentement général fait son apparition dans deux formulaires du Service de transfusion CRS Suisse - le Consentement du donneur à s'inscrire dans le registre de la Fondation Cellules souches du sang et le Consentement du donneur pour des tests complémentaires - où des enjeux liés à l'utilisation à des fins de recherche des échantillons sont abordés. Par exemple, dans le premier formulaire on peut lire l'énoncé suivant:

«J'accepte que du sang (ou ses composantes), prélevé pour le groupage HLA soit conservé et utilisé pour des tests en vue de déterminer mon éventuelle compatibilité avec un patient donné, ou dans l'intérêt de la recherche dans le domaine de la transplantation et de la variabilité génétique des populations».

Nous pouvons constater que deux règles de consentement (éclairé et général) coexistent dans le même formulaire. Cette coexistence soulève plusieurs remarques. Premièrement, le consentement général introduit un assouplissement dans l'un des éléments centraux du consentement éclairé, c'est-à-dire ces «éléments d'information» (disclosure et understanding) qui, avec les «éléments de seuils» (competence et volontariness) constituent les éléments de base du consentement éclairé (informed consent) [4]. Cet assouplissement n’est pas anodin parce que le consentement éclairé (ou informé) du donneur, comme manifestation concrète du droit à l'auto-détermination [5], constitue la pierre angulaire du dispositif normatif qui a été mis en place au niveau national et international [6]. Sur ce point, il faut également observer que le Service de transfusion CRS Suisse circonscrit et limite les domaines de recherche à la transplantation et à la variabilité génétique des populations, et cet élément peut être considéré comme un élément d'information (même si partiel). Cette limitation implique cependant que des projets de recherche futurs ayant d'autres objectifs ne rentrent pas dans le cadre défini par ces formulaires de consentement. Deuxièmement, il est délicat d'associer dans le même formulaire deux demandes avec des objectifs différents. En effet, même si le donneur peut biffer les énoncés concernant la recherche (ce qui arrive dans un certain nombre de cas), les deux énoncés étant si étroitement liés, le donneur pourrait involontairement être amené à penser que les deux objectifs sont indissociables. Il serait ainsi préférable de séparer les deux demandes, afin de rendre plus manifeste qu'il s'agit bien de deux décisions et de deux consentements différents. Troisièmement, les trois justifications au consentement général qui reviennent le plus souvent dans la littérature - à savoir 1) une manière de réduire les coûts et les difficultés associés à la nécessité de recontacter des milliers de donneurs, 2) la priorité donnée à l'intérêt public dans la réalisation de projets de recherche, et 3) le fait que les recherches sur ces échantillons ne représentent qu'un risque minimal pour les donneurs - bien que recevables, sont loin de susciter l'adhésion [7] et méritent partant une analyse plus poussée, notamment parce que la pérennité de la HSCT repose sur la confiance des donneurs dans les institutions en charge de celle-ci, confiance qui pourrait être mise à mal si le respect de principes éthiques fondamentaux était perçu comme insuffisant.

Or, si le consentement éclairé ne représente pas dans le cas en espèce une solution pratique et si le consentement général ne donne pas encore de garanties suffisantes, comment procéder?

Nous sommes de l'avis qu'il faudrait rester - dans la mesure où un équilibre raisonnable entre l'intérêt privé du donneur et l'intérêt légitime de la recherche est réalisable - au plus près du standard du consentement éclairé; le donneur devrait en ce sens pouvoir choisir le régime de consentement (éclairé ou général) auquel il est disposé à se soumettre par rapport à l'utilisation future de matériel biologique humain dans le cadre de la recherche, et il devrait pouvoir se déterminer spécifiquement sur ce point, autrement dit séparément de toute utilisation à des fins diagnostiques ou thérapeutiques des échantillons comme nous l'avons souligné auparavant. Il faudrait bien entendu encore tester la praticabilité de cette solution. Si cela se révélait difficile, une alternative pourrait consister dans le fait d'assigner à une commission d'éthique de la recherche la tâche d'évaluer, le moment venu, si un protocole de recherche déterminé satisfait les principes éthiques en vigueur et respecte les intérêts légitimes du donneur, sans que celui-ci se détermine expressément sur l'utilisation des échantillons.

\section{Correspondance \\ Dr Jörg Halter}

Universitätsspital Basel

Abteilung Hämatologie

Spitalstrasse 21/Petersgraben 4

$\mathrm{CH}-4031$ Base

E-mail: jhalter[at]hin.ch

\section{Références}

1. Shaw BE, et al. Donor Safety: The Role of the WMDA in Ensuring the Safety of Volunteer Unrelated Donors: Clinical and ethical considerations. Bone Marrow Transplantation. 2010;45(5):832-8.

2. King RJ, et al. Unrelated hematopoietic stem cell donors as research subjects. Bone Marrow Transplantation. 2011;46(1):10-3.

3. Académie Suisse des Sciences Médicales: Biobanques: Prélèvement, conservation et utilisation de matériel biologique humain. 2006

4. Beauchamp TL, Childress JF. Principles of Biomedical Ethics. 6th edition. Oxford/New York: University Press; 2009. p. 118-40.

5. Académie Suisse des Sciences Médicales: Droits des patientes et patients à l'autodétermination. 2005; Report of the International Bioethics Committee of UNESCO (IBC) on Consent. 2007.

6. Loi sur la transplantation (RS 810.21); WHO Guiding Principles on Human Cell, Tissue and Organ Transplantation. Transplantation. 2010;90(3):229-33, voir notamment le principe directeur $n^{\circ} 3$ et le commentaire qui l'accompagne.

7. Caulfield T, Kaye J. Broad consent in biobanking: Reflections on seemingly insurmountable dilemmas. Medical Law International. 2009;10:85-100. 